\title{
Anemia arregenerativa en el lactante: 2 casos de síndrome de Pearson
}

\author{
A regenerative anemia in infants: 2 cases of Pearson's syndrome
}

\author{
Lic. José M. Martínez de Zabarte Fernández, ${ }^{a}$ Lic. Carmen Rodríguez-Vigil Iturrate, Lic. Cristina Martínez Faci, \\ Lic. Inmaculada García Jiménez, ${ }^{c}$ Lic. Laura Murillo Sanjuan ${ }^{a}$ y Lic. Ascensión Muñoz Mellado ${ }^{b}$
}

\section{RESUMEN}

La anemia es frecuente en lactantes, $\mathrm{y}$, aunque su causa, habitualmente, es banal, debe establecerse un diagnóstico etiológico adecuado. Cuando la anemia es arregenerativa, puede deberse a aplasia medular, síndrome mielodisplásico, infiltración medular o déficits de factores hematopoyéticos. Otra posible causa es el síndrome de Pearson, una rara enfermedad mitocondrial que se presenta con anemia asociada a otras citopenias, insuficiencia pancreática, acidosis láctica y gran variabilidad en su presentación clínica condicionada por la heteroplasmia. Escaracterístico encontraren el aspirado/biopsia de médula ósea vacuolización de los precursores de serie roja y sideroblastos en anillo. El diagnóstico de certeza se establece mediante el estudio genético del ácido desoxirribonucleico mitocondrial con Southern blot (amplificación completa deácido desoxirribonucleico mitocondrial mediante reacción en cadena de la polimerasa-largo), que obtiene deleción del 70\%-80\% de $4977 \mathrm{pb}$ (DNAm 8343-13459). No existe tratamiento curativo y las medidas son de soporte. Es frecuente el fallecimiento en los primeros años de vida.

Se presentan dos casos de lactantes con síndrome de Pearson. Palabras clave: lactante, anemia, anemia arregenerativa, enfermedades genéticas innatas, síndrome de Pearson.

\section{ABSTRACT}

Anemia is very common in infants. Although its causes are usually not severe and treatable, proper etiologic diagnosis should be established. When anemia is non-regenerative, it can be caused by aplastic anemia, myelodysplastic syndrome, bone marrow infiltration or hematopoietic factors deficiencies.

a. Servicio de Pediatría, Hospital Universitario Miguel Servet. Zaragoza. España.

b. Unidad de Oncohematología Pediátrica, Servicio de Pediatría, Hospital Universitario Miguel Servet. Zaragoza. España.

c. Unidad de Enfermedades Metabólicas, Servicio de Pediatría, Hospital Universitario Miguel Servet.

Zaragoza. España.

Correspondencia:

Lic. José M. Martínez de Zabarte Fernández, chemi87@hotmail.com

Financiamiento: Ninguno.

Conflicto de intereses: Ninguno que declarar.

Recibido: 1-5-2016

Aceptado: 12-8-2016
Another possible cause is Pearson's syndrome, a rare mitochondrial disease that causes non-regenerative anemia associated with other cytopenias, pancreatic insufficiency, lactic acidosis and great variability in clinical presentation conditioned by heteroplasmy. It is characteristic to find in bone marrow studies variable vacuolization in erythroblastic progenitors and ring sideroblasts. The diagnosis is established by genetic study of mitochondrial deoxyribonucleic acid performed by Southern blot analysis (complete mitochondrial deoxyribonucleic acid amplification by polymerase chain reaction -long), obtaining $70-80 \%$ deletion of $4977 \mathrm{bp}$ (NMD 8343-13459). There is no curative therapy and support treatment is the only available nowadays. Death is frequent in early years of life.

Two cases of infants with Pearson syndrome are presented. Key words: infant, anemia, aregenerative anemia, genetic diseases inborn, Pearson syndrome.

http:/ /dx.doi.org/10.5546/aap.2017.e24

\section{INTRODUCCIÓN}

La anemia es un trastorno frecuente y, por lo general, benigno en el lactante. Su causa más usual es la ferropenia por carencia de aporte nutricional, que presenta una prevalencia de $9,6 \%-35 \%$ en menores de 24 meses. ${ }^{1,2}$

La anemia puede ser el signo inicial de una patología de mayor gravedad. Por ello, debe estudiarse siempre su etiología.

Se presentan dos casos de anemia en lactantes, en los que se llegó a confirmar el diagnóstico de una patología muy poco frecuente.

\section{CASOS CLÍNICOS \\ Caso 1}

Lactante varón de 8 meses sin antecedentes de interés, hospitalizado por presentar anemia arregenerativa en la analítica sanguínea realizada en el contexto de cuadro febril, secundario a una infección respiratoria (hemograma, en la Tabla 1). Se obtuvo una muestra de secreciones respiratorias mediante aspirado nasofaríngeo y se realizó inmunofluorescencia directa con anticuerpos monoclonales específicos, que fue positiva para metapneumovirus humano.

Se realizó un estudio etiológico de la anemia (bioquímica con deshidrogenasa láctica 
-lactic dehydrogenase; LDH, por sus siglas en inglés-, haptoglobina, metabolismo del hierro, vitamina B12, ácido fólico, Coombs directa), con resultados normales. En el estudio de médula ósea realizado durante su ingreso, se observó dishemopoyesis de las 3 series: vacuolización en serie roja con sideroblastos "en anillo" en tinción de Pearls y discreta dismorfia megacariocítica y granulocítica sin cumplir criterios de síndrome mielodisplásico. Durante el seguimiento, presentó progresión hematológica a pancitopenia, con requerimientos transfusionales de hematíes periódicos y repetidas infecciones que motivaron múltiples ingresos hospitalarios. La ganancia ponderoestatural fue estable durante el seguimiento con longitud en P25 y peso en P50. Se realizó el estudio genético de anemia de Blackfan-Diamond y de hemoglobinuria paroxística nocturna, que fueron negativos. Con 2 años y 3 meses, ante la persistencia de la sintomatología, se decidió realizar un estudio genético del ácido desoxirribonucleico (ADN) mitocondrial con Southern blot, a través de la amplificación completa de ADN mitocondrial mediante PCR-largo, que obtuvo deleción en torno al 70\%-80\% de 4977pb (DNAm 8343-13 459) y confirmó el diagnóstico de síndrome de Pearson. No se han encontrado alteraciones en otros órganos hasta el momento.

\section{Caso 2}

Lactante varón de 8 meses sin antecedentes de interés, que ingresó por fiebre sin foco aparente, palidez, taquicardia y petequias pretibiales. En la analítica inicial, presentaba anemia arregenerativa, trombopenia y neutropenia moderada (Tabla 1). En el estudio microbiológico realizado, se encontró un resultado serológico positivo para inmunoglobulina $\mathrm{M}$ (IgM) de Mycoplasma pneumoniae.

Tabla 1. Datos hematológicos iniciales en ambos pacientes

\begin{tabular}{|c|c|c|}
\hline & Caso 1 & Caso 2 \\
\hline Leucocitos $/ \mathrm{mm}^{3}$ & 10800 & 3500 \\
\hline Neutrófilos totales $/ \mathrm{mm}^{3}$ & 2280 & 400 \\
\hline Hemoglobina (g/dl) & 3,6 & 3,5 \\
\hline Hematocrito & $9,9 \%$ & $9,2 \%$ \\
\hline Reticulocitos (*103/ul) & 13,05 & 6,55 \\
\hline Reticulocitos (\%) & $0,61 \%$ & $0,43 \%$ \\
\hline VCM (fL) & 103 & 91 \\
\hline Plaquetas $/ \mathrm{mm}^{3}$ & 186000 & 25000 \\
\hline
\end{tabular}

VCM: volumen corpuscular medio.
Se realizó un estudio analítico para determinar la etiología de la pancitopenia (bioquímica, metabolismo del hierro, vitamina B12, ácido fólico, Coombs directa, anticuerpos antigranulocito), con resultados normales. El estudio de médula ósea presentó marcada hipoplasia de serie roja; no se realizó tinción de Pearls. Necesitó transfusión de hematíes y plaquetas repetidamente.

Se observaron deposiciones dispépticas con restos alimenticios sugestivos de insuficiencia pancreática. En la ecografía abdominal, presentaba hiperecogenicidad pancreática inespecífica. La elastasa en heces fue normal. Presentaba ganancia ponderoestatural adecuada desde el nacimiento, con peso y longitud mantenidos en P15. Aunque la médula ósea era más compatible con anemia de Blackfan-Diamond, la clínica sugestiva de insuficiencia pancreática motivó, a los 9 meses de edad, la realización del estudio genético del ADN mitocondrial con Southern blot, que obtuvo deleción en torno al 70\%-80\% de $4977 \mathrm{pb}$ (DNAm 8343-13459) y confirmó el diagnóstico de síndrome de Pearson. Se inició tratamiento con coenzima Q10, ácido fólico y vitamina C.

\section{DISCUSIÓN}

El síndrome de Pearson es una rara enfermedad mitocondrial descrita por primera vez en 1979 en un paciente con anemia arregenerativa, vacuolización en serie roja e insuficiencia pancreática. ${ }^{3} \mathrm{La}$ incidencia no está claramente establecida, pero podría situarse en torno a $1,6 / 10^{5}$ habitantes. ${ }^{4}$ Consiste en una alteración del ADN mitocondrial, que produce un mal funcionamiento de la cadena respiratoria que se lleva a cabo en la mitocondria. ${ }^{5}$

Habitualmente, comienza con anemia arregenerativa asociada o no a otras citopenias, que suelen aparecer durante el seguimiento.,4

La clínica inicial puede ser muy variada; dependerá del grado de afectación de los diferentes órganos. Esta afectación heterogénea está condicionada por la heteroplasmia: las alteraciones genéticas del ADN mitocondrial están presentes de forma variable en los diferentes tejidos y órganos, y tienen un grado distinto de afectación dependiendo del número de mitocondrias con material genético alterado y la función que estas realicen en el órgano o tejido.,6

Un $20 \%-80 \%$ de los pacientes presentan insuficiencia pancreática ${ }^{6,7}$ Este diagnóstico se sospechó en el caso 2 por las características dispépticas de las deposiciones, sin poder establecerse el diagnóstico. Otras manifestaciones 
clínicas que pueden darse en el síndrome de Pearson se enumeran en la Tabla $2 .{ }^{8}$

El cuadro hematológico tiende a mejorar durante los primeros años, y adquieren mayor importancia otras manifestaciones clínicas que pueden aparecer progresivamente. Los casos que superan los 3 años de edad pueden desarrollar un síndrome de Kearns-Sayre (oftalmoplejia refractaria, retinitis pigmentaria y, ocasionalmente, ataxia, bloqueo cardíaco e hiperproteinorraquia). ${ }^{4,9}$ En ocasiones, el síndrome de Pearson puede derivar en síndrome de Leigh. ${ }^{10}$

El diagnóstico diferencial, especialmente al inicio del cuadro, se realiza con otras anemias arregenerativas y con síndromes mielodisplásicos, aunque deben excluirse las causas de anemia más frecuentes, como las carenciales. Para este estudio, debe realizarse una analítica sanguínea: estudio bioquímico básico con ionograma, medición de lactato y piruvato, gasometría, metabolismo del hierro, vitamina B12 y ácido fólico, Coombs directa. En ocasiones, debe descartarse la hemoglobinuria paroxística nocturna, habitualmente, mediante citometría de flujo.

Es necesario realizar el estudio histopatológico de médula ósea mediante aspirado y/o punción ósea, en el que es característico encontrar vacuolización de precursores de serie roja y sideroblastos en anillo. ${ }^{3}$ Es frecuente la duda diagnóstica con el síndrome mielodisplásico. ${ }^{6}$

También resulta frecuente la confusión diagnóstica con la anemia de Blackfan-

TABLA 2. Manifestaciones clínicas y complicaciones en el sindrome de Pearson

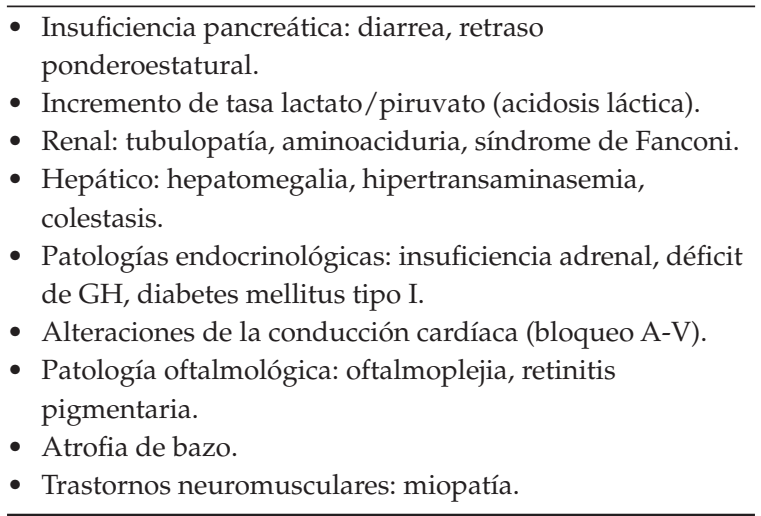

GH: hormona de crecimiento; bloqueo A-V: bloqueo auriculoventricular.
Diamond. En una revisión de una cohorte de 362 pacientes con diagnóstico establecido de anemia de Blackfan-Diamond en los que se pretendía encontrar posibles pacientes afectos de síndrome de Pearson que hubieran sido diagnosticados erróneamente, 8 de ellos (2,2\%) fueron, finalmente, diagnosticados con este síndrome. ${ }^{11}$ También debe tenerse en cuenta, en el diagnóstico diferencial, el síndrome de Shwachman-Diamond, que presenta insuficiencia pancreática exocrina y disfunción de médula ósea. ${ }^{12}$

El diagnóstico de certeza se realiza mediante el estudio genético del ADN mitocondrial con Southern blot y amplificación completa de ADN mitocondrial mediante PCR-largo. Suele encontrarse una deleción de ADN mitocondrial de, aproximadamente, $70 \%-80 \%$; la más frecuente es de alrededor de $4977 \mathrm{pb}$ (ADN mitocondrial 8343-13459), que afecta a múltiples genes, como ocurre en nuestros pacientes. ${ }^{4}$ Otras alteraciones del ADN mitocondrial permiten establecer el diagnóstico del síndrome de Pearson, como la deleción de 5712 pb de ADN mitocondrial, que afecta a los nucleóticos 8011-13 722.13 No parece existir relación entre el número de pares de bases afectados y la localización de estos en el genoma con el tipo de afectación clínica y la evolución. ${ }^{8}$

No existe, actualmente, un tratamiento curativo y es frecuente el fallecimiento de estos pacientes (hasta un 50\%) en los primeros 3 años de vida ${ }^{7,11}$ a consecuencia de infecciones y complicaciones asociadas a las graves citopenias. ${ }^{13}$ A partir de esta edad, el cuadro hematológico tiende a mejorar y la mortalidad se debe a otras complicaciones que presenta el síndrome de Pearson (Tabla 2).

Durante la etapa de mayor afectación medular ósea, es usual que el paciente necesite soporte transfusional frecuente. ${ }^{8}$ Una opción terapéutica es el trasplante alogénico de precursores hematopoyéticos, aunque este tratamiento no modifica las manifestaciones extrahematológicas de esta enfermedad. ${ }^{4}$

Se han publicado recientemente 4 casos de pacientes con síndrome de Pearson con infección bacteriana asociada a neutropenia sin respuesta a la antibioterapia intravenosa y que recibieron tratamiento con factor estimulante de colonias de granulocitos durante el proceso infeccioso; dos de ellos presentaron buena evolución, ${ }^{8,14}$ por lo que podría plantearse esta medida en aquellos pacientes con infecciones graves y con evolución tórpida. 
Se ha intentado el tratamiento de la anemia con eritropoyetina, sin respuesta satisfactoria. ${ }^{8}$

Existen casos con mejoría de la anemia al suplirles coenzima Q10. ${ }^{6}$ También se han empleado complejos vitamínicos del grupo B, especialmente con vitamina B12 y ácido fólico, para mejorar la evolución de la anemia. ${ }^{15}$ No existe evidencia suficiente para establecer un claro beneficio al recibir estos tratamientos.

Como conclusión, el síndrome de Pearson es una patología muy desconocida por los facultativos, probablemente debido a su escasa prevalencia. Esto lo convierte en una entidad infradiagnosticada, ya que su presentación clínica es muy variada y, en el estudio etiológico, es fácilmente confundible con otras patologías. Debe tenerse siempre en cuenta la sospecha en aquellos lactantes que presenten una anemia arregenerativa, especialmente con signos de vacuolización en el estudio de médula ósea.

\section{REFERENCIAS}

1. Moráis López A, Dalmau Serra J, Gil Campos M, Martínez Suárez V, et al. Importancia de la ferropenia en el niño pequeño: Repercusiones y prevención. An Pediatr 2011;74(6):415.e1-10.

2. Donato H, Cedola A, Rapetti MC, Buys MC, et al. Anemia ferropénica: Guía de diagnóstico y tratamiento. Arch Argent Pediatr 2009;107(4):353-61.

3. Pearson HA, Lobel JS, Kocoshis SA, Naiman JL, et al. A new syndrome of refractory sideroblastic anemia with vacuolization of marrow precursors and exocrine pancreatic dysfunction. J Pediatr 1979;95(6):976-84.

4. Tumino M, Meli C, Farruggia P, La Spina M, et al. Clinical Manifestations and Management of Four Children With Pearson Syndrome. Am JMed Genet A 2011;155A(12):3063-6.
5. Guirado Giménez F, Montoya Villarroya JA, Oliván del Cacho MJ,Playán Ariso A, et al. Paciente con síndrome de Pearson y de Kearns-Sayre y la deleción común de $4,9 \mathrm{~Kb}$ del ADN mitocondrial en sangre. An Esp Pediatr 1998;49(5):510-2.

6. Martín Hernández E, García Silva MT, Quijada Fraile P, Martínez de Aragón A, et al. Síndromes de Pearson y de Kearns-Sayre: dos enfermedades mitocondriales multisistémicas, debidas a deleciones en el ADN mitocondrial. Acta Pediatr Esp 2010;68(9):451-9.

7. Rötig A, Bourgeron T, Chretien D, Rustin P, etal.Spectrum of mitochondrial DNA rearrangements in the Pearson marrowpancreas syndrome. Hum Mol Genet 1995;4(8):1327-30.

8. Farruggia P, Di Cataldo A, Pinto RM, Palmisani E, et al. Pearson Syndrome: A Retrospective Cohort Study from the Marrow Failure Study Group of A.I.E.O.P. (Associazione Italiana Emato-Oncologia Pediatrica). JIMD Rep 2016;26: 37-43.

9. Kearns TP, Sayre GP. Retinitis pigmentosa, external ophthalmophegia, and complete heart block: unusual syndrome with histologic study in one of two cases. AMA Arch Ophthalmol 1958;60(2):280-9.

10. Santorelli FM, Barmada MA, Pons R, Zhang LL, etal. Leightypeneuropathology in Pearson syndrome associated with impaired ATP production and a novel mtDNA deletion. Neurology 1996;47(5):1320-3.

11. Alter BP. Pearson syndrome in a anemia cohort. Blood 2014;124(3):312-3.

12. MacipeCosta R,Javierre Miranda E,Lou Francés M,Heredia González S, et al. Síndrome de Shwachman-Diamond. An Pediatr 2006;65(1):79-82.

13. Park J, Ryu H, Jang W, Chae H, et al. Novel 5.712 kb mitochondrial DNA deletion in a patient with Pearson syndrome: a case report. Mol Med Rep 2015;11(5):3741-5.

14. Baertling F, Meissner T, Troeger A, Pillekamp F, et al. Granulocyte colony stimulating factor for treatment of neutropenia-associated infection in Pearson syndrome. Klin Padiatr 2014;226(3):190-1.

15. Topalo lu R, Lebre AS, Demirkaya E, Ku konmaz B, et al. Two new cases with Pearson syndrome and review of Hacettepe experience. Turk J Pediatr 2008;50(6):572-6. 